\title{
APORTES DE LA CRÍTICA GENÉTICA A LOS ESTUDIOS COMPARADOS: REFLEXIONES METODOLÓGICAS A PARTIR DE AMADEUS, DE PETER SHAFFER
}

\author{
Lucas GAGLIARDI
}

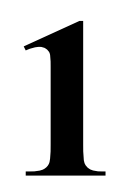

\section{Introducción}

Los estudios comparativos llevan más de un siglo de reflexiones, incluyendo el cuestionamiento a su propio estatuto y objeto de debate. Estas revisiones - como por ejemplo las de René Wellek (1956) - han conllevado a la evaluación de escuelas y tradiciones críticas al respecto, así como a examinar nuevamente aquellos temas de larga data para el comparatismo: cuestiones relacionadas con las tradiciones nacionales, la universalidad de algunos hechos literarios, el diálogo entre diferentes lenguajes, las fuentes, la traducción, por nombrar solo algunas cuestiones (Muñiz, 1989).

Por otro lado, en las últimas décadas, los avances en los estudios sobre los procesos creativos han aportado una serie de reflexiones de interés para el desarrollo de los estudios comparativos. En particular, me interesa plantear un diálogo entre el comparatismo tradicionalmente definido y un enfoque que proviene de la filología: la genética textual, también llamada crítica genética. Esta mirada sobre la literatura, según creo, puede proveer observaciones metodológicas relevantes dado que plantea una concepción muy particular de la obra literaria con consecuencias que pueden enriquecer el trabajo del crítico. Como señala Muñiz (1989), existen corrientes dentro del comparatismo que se orientan al trabajo con la poética y la génesis de las obras - aunque estas últimas han sido menos desarrolladas o desestimadas por autores como Fokkema-; a partir de esta situación históricamente, proponemos acercar nuevamente al comparatismo y a los estudios de la génesis literaria. Recurriremos, por un lado, a una sucinta exposición de los principios teóricos y metodológicos de la genética textual; por otro, analizaremos un caso particular: el proceso escritural de la pieza teatral Amadeus, del dramaturgo inglés Peter Shaffer, que es objeto de investigaciones que desarrollo actualmente. Amadeus, estrenada en 1979 en el West End de Londres, posee un recorrido textual de diferentes versiones publicadas bajo las revisiones constantes de su autor. De estas versiones extraeremos algunos problemas o ejes de reflexión que permitirán plantear los aportes de la genética al comparatismo. 


\section{Marco metodológico de la genética textual}

Como señala Élida Lois (2001: 6-7; 2005a; 2005b), una de los referentes de la genética textual en Argentina, esta perspectiva tiene su origen a mediados del siglo pasado en Francia, aunque es posible hallar algunos precursores anteriores. Esta corriente surge como una forma de ruptura con respecto a la filología de raigambre lachmaniana (Lois, 2005a: 50) y a raíz de las diferencias observables en cuanto a condiciones materiales de los procesos genéticos a lo largo de la historia: el estudio filológico de textos clásicos o medievales presenta materiales diferentes al manuscrito moderno, posterior a la invención de la imprenta; por ende, este último requerirá consideraciones particulares. Se vuelve así posible una reflexión sobre el estatuto de la escritura y la obra que postula su diferencia para con las condiciones de producción precedentes a la modernidad (Lois, 2005a: 51$52)$.

La primera distinción relevante de la crítica genética para con otras perspectivas dentro de la disciplina denominada «filología» consiste en prestar atención al proceso de escritura en todas sus instancias, intentando no entronizar un estadio textual como el más puro, legítimo o valioso. Se intenta evitar una aproximación teleológica a la escritura (Lois, 2001: 17; 2005b: 86-87). Esto es consecuencia de que el interés de esta perspectiva no sea el de editar el texto más cercano posible al arquetipo textual -objetivo de la ecdótica, por ejemplo- sino interpretar lo que ha ocurrido en el transcurso de la escritura: qué ha puesto en juego el autor, cuáles fueron sus planes, sus cambios, sus operaciones, sus conflictos. En este sentido, esta perspectiva se encuentra más cerca de lo que Werner Hamacher entiende por filología:

La filología no es una teoría en el sentido de una inspección en aquello que es. Tampoco es una práctica que se deriva de una teoría o que encuentra su fin en una teoría. Cuando es, es el movimiento del atender a aquello con lo que se encuentra este atender y que lo desvía, lo acomete y erra, aquello que lo atrae y que atrayéndolo de ese modo se aleja de él. Es la experiencia de la tracción en la sustracción. El movimiento de una búsqueda sin un fin predeterminado. Por lo tanto, sin fin. Por lo tanto, sin el «sin» de un fin. Sin el «sin» de la ontología (Hamacher, 2001: 13).

La genética textual considera que la escritura es inherentemente recursiva y susceptible de ser activada y reactivada en cualquier momento. Debido a esta concepción, conceptos como los de «pretextos pre-redaccionales» y «redaccionales» se muestran como categorías que describen las funciones de una porción de escritura dentro del proceso y dentro del archivo que conforman (Goldchlluk, 2009; 2010), sin implicar una sucesión temporal ni describir la naturaleza material de los documentos. Dicho de otro modo: un documento que contiene un estadio textual puede ser «redefinido». De este modo, el manuscrito — objeto privilegiado por la genética一 no es el único tipo de documento que puede funcionar como pre-texto. Un texto édito, a su vez, puede ser abordado como pre-texto redaccional cuando una nueva edición supervisada por el autor sea publicada. En el caso particular de la génesis de Amadeus, esta consideración resulta fundamental: dado que no se cuenta con los manuscritos de su autor, se ha trabajado con las distintas versiones publicadas con revisiones y comentarios del propio Peter Shaffer para la constitución del dossier genético. 
Estas nociones nos llevan a dos consideraciones cruciales para esta perspectiva y, por extensión, para el comparatismo: ¿Dónde comienza y termina la obra? ¿Qué es el autor? En cuanto a la primera, la genética desarticula la equiparación de la obra con el texto édito. Las reflexiones del geneticismo desarrolladas en Argentina (principalmente las de Lois y los grupos de investigación dirigidos por Graciela Goldchluk) han postulado que la obra es, antes que la mera suma de estadios textuales, aquello que acontece entre esas diferentes instancias; de hecho, teóricos como el francés Louis Hay han llegado a afirmar que «el texto no existe», entendiendo por «texto» una fijación de la obra (Hay, 1996: 7). En cuanto al autor, esta perspectiva evita postularse a sí misma como una nueva psicología del autor en tanto recurre a la noción derrideana de «firma» (Derrida, 1971); se trabaja con un sujeto real que escribe, eso es cierto, pero lo que conocemos del mismo es en función de su escritura, la imagen de autor que esta nos brinda, y otros datos que podemos conocer sobre el mismo a través de otras fuentes. Aunque Lois (2001; 2005a; 2005b) reconozca aportes del psicoanálisis a la genética, esto no significa indagar en el inconsciente de un autor a través de su escritura. Es por todo esto también por lo que en nuestro recorte tenemos en cuenta las versiones del texto teatral publicadas con revisiones de Shaffer y el guión cinematográfico que este último firma, no así la película en su conjunto que reviste una serie de lenguajes y operaciones de las cuales Shaffer no es responsable directo ${ }^{1}$.

Como se observa, estos planteos tienen consecuencias para la tarea del crítico: suponen pensar con detenimiento en el recorte al momento de la construcción de un corpus, dado que los bordes que definirían a la obra parecen desdibujarse. En este sentido, Goldchluk (2010) se pregunta «¿Dónde sucede la literatura?», poniendo en evidencia que la misma es un acontecimiento y una experiencia antes que una «cosa» delimitada en un protocolo escrito, puntualmente, en el formato que hoy identificamos con el libro. A su vez, estas consideraciones llevan a repensar cómo se concebirá la intertextualidad, como veremos más adelante.

Observamos entonces una serie de afinidades entre el trabajo genético y el comparatista. Dado que nuestro enfoque trabaja con variantes textuales, implica el cotejo; a su vez, para la indagación en la génesis de un texto, será necesario tener en cuenta los elementos de aquellos que «rodea» y de entrelaza con la obra, aquello que Marc Angenot denomina «discurso social»: la totalidad de lo decible y escribible en un contexto, regulado por una hegemonía (Angenot, 2010: 25) ${ }^{2}$. Por otra parte, leer variantes textuales supone enfrentarse a problemas fluctuantes, que aparecen y reaparecen suponiendo «conflictos discursivos» (Lois, 2005a: 52) en el trabajo del autor; el análisis de estos últimos constituye uno de los principales puntos de indagación de la genética. Se vuelve posible leer entre líneas y generar microcircuitos de interpretación dentro del proceso escritural. La posibilidad de leer estos puntos (sometidos a fluctuaciones) es lo que llamaremos, junto con Rasic (2013), «lectura constelada», en tanto nos permite agrupar elementos o puntos dentro de la génesis escritural de la obra y efectuar una

\footnotetext{
${ }^{1}$ Esto no implica que no pueda realizarse un análisis comparativo del guión y su realización concreta en el filme de Milos Forman. A su vez, pueden realizarse observaciones interesantes sobre las elecciones y recursos de la película y su diálogo con la obra de Shaffer, pero resultaría necesario prescindir del concepto de firma y des-centrar el proceso escritural como lo concibe la genética.

${ }^{2}$ La crítica genética desarrolla esta idea desde el concepto de sociogénesis referido en Lois (2001; 2005a; 2005b).
} 
interpretación que es a la vez personal y basada en evidencia. Esta figura de lectura, que cree en la posibilidad de conexiones múltiples y multidireccionales puede compararse con la figura del rizoma propuesta por Giles Deleuze y Félix Guattari en Mil mesetas (1988).

A continuación, nos aproximaremos a Amadeus a través de conceptos como el de intertextualidad y el análisis de motivos a lo largo de la génesis. Para las transcripciones sinópticas, utilizamos las siguientes convenciones en cuanto a la delimitación de las versiones del texto:

André Deutsc, 1981 (versión 1);

Harper Collins, 1984 (Versión 2);

Guión cinematográfico, 1983 (versión 3);

Harper Perennial, 2001 (versión 4).

Para referencias bibliográficas detalladas, remitimos a la bibliografía al final de este artículo.

\section{Amadeus: la reescritura y sus afectaciones}

a) Problematización del concepto de intertextualidad. La presencia de intertextos en una obra ha sido discutida una y otra vez, así como las cuestiones de la influencia y la «originalidad». Desde el mentado concepto de «fuente» a las ideas que propone el geneticismo hay un largo trecho: mientras que las fuentes suponen una suerte de acervo del cual el autor extrae o un acervo que se vuelca unidireccionalmente sobre el autor pasivo (en forma teleológica), la genética elige regresar a la idea de la bivocalidad de la palabra propuesta por Mijaíl Bajtín (1988: 254): el acento se pondrá en el rol activo del escritor en la selección, apropiación y transformación de esa palabra ajena. Recordemos que el teórico ruso llegó a noción de bivocalidad y de dialoguismo entre otras, no la de intertextualidad —una formulación posterior de Julia Kristeva y Roland Barthes ${ }^{3}$ —; por lo tanto, cuando utilizamos el término «intertexto» - al cual no renunciamos dado su fuerte arraigo en la teoría y crítica literarianos referiremos en realidad al enunciado ajeno con el cual la palabra del autor establece un diálogo. La figura del palimpsesto resulta pertinente aquí: no se trata tanto de reescribir sino de escribir sobre la escritura anterior y el modo en que las huellas de una se sienten sobre la otra; de este modo, el texto nuevo afecta también al que lo precede y evita la unidireccionalidad del concepto de fuentes. Formulaciones posteriores, como la de Michael Rifaterre proponen incluso que la intertextualidad no está inscripta en el texto sino que resulta un efecto de lectura (Ferrer, 2007), lo cual autores como Ferrer relativizan. De ser así ¿cómo explicaríamos la intertextualidad dentro de la génesis, que es un fenómeno de escritura? El primer problema que induce a un replanteo de la intertextualidad es determinar qué condiciones hacen que un texto ajeno sea reconocible dentro del texto analizado. ¿Existen siempre señalizaciones? ¿Cómo determinar si un autor está o no trabajando sobre la base de un intertexto? ¿Lo hace en forma consciente o inconsciente?

¿Sería necesario, entonces, prescindir por completo de la noción de intertextualidad cuando nos interesamos en la génesis? Ciertamente no, ya que para apropiarse de la noción basta con recordar que todo

\footnotetext{
${ }^{3}$ Al respecto, pueden consultarse los trabajos de Patrick Sériot (2003) e Iván Villalobos Alpízar (2003).
} 
Aportes de la crítica genética a los estudios comparados: reflexiones metodológicas...

escritor es también un lector, que todo gesto de escritura constituye también un gesto de lectura. Tanto más si se tiene en cuenta que, contrariamente a la tradicional crítica de las fuentes (que, por otro lado, generalmente no se complica recurriendo a los manuscritos), la crítica genética se preocupa menos de designar un origen que de analizar lo que constituye la esencia misma de la intertextualidad: el trabajo de asimilación y transformación que caracteriza todo proceso intertextual (Ferrer, 2007).

En cuanto a Amadeus, la crítica ha comparado esta obra con Mozart y Salieri $(1830)^{4}$, de Alexander Pushkin, que fuera adaptada posteriormente por Nikolas Rimsky-Korsakov (1898) en forma de ópera. En entrevistas que Peter Shaffer brindó durante las primeras producciones de la obra y en declaraciones posteriores, él afirmaba desconocer el texto de Pushkin (Borowitz, 2005: 140); si seguimos estas declaraciones, la pieza del escritor ruso no sería un enunciado que Shaffer hubiese tenido en consideración ya sea para escribir en consonancia o en contra del mismo; dicho de otro modo, Amadeus no tendría una relación intertextual consciente con la pieza de Pushkin. Al no contar con pretextos pre-redaccionales, ¿cómo determinamos la presencia y la diferencia de este intertexto en Shaffer? Y más importante aún ¿cuáles serían las inflexiones particulares de Shaffer y su obra en cuanto al tema de la rivalidad Mozart-Salieri? ¿Cómo releemos a Pushkin después de Amadeus? Para el geneticismo, cada acto de escritura implica una diferencia, de acuerdo con las ideas de Derrida (1998a) que considera el acto de escribir como un acto de diferencia (diferenciación de otra cosa; diferir en el tiempo) ${ }^{5}$; rastrearemos tanto el posible contacto con Pushkin y las retroalimentaciones entre ambas obras.

Pushkin nos muestra una representación de Wolfgang Mozart muy difundida durante el Romanticismo: la del músico como encarnación del genio visionario romántico; su carácter de «libertino» y el envenenamiento perpetrado por un envidioso Salieri. El escritor ruso recoge rumores que, durante los últimos años de vida de Salieri, habían cimentado esa leyenda y esboza una primera oposición relevante en su texto: al Mozart libre y jubiloso se le opone un Salieri que aprendió música «diseccionándola» o tratándola como si de «álgebra»se tratase (Pushkin, 1942: 45) . En el contexto del Romanticismo tardío, esta oposición entre formas dictadas por la pasión ${ }^{7}$ y las dictadas por principios de simetría, equilibrio y racionalidad resulta de lo más común; no obstante, aunque Shaffer muestra algunas similitudes iniciales, el tratamiento resulta completamente nuevo.

\footnotetext{
${ }^{4}$ Aunque fuera terminada en esta fecha, existen datos de que Pushkin comenzó a escribirla en 1826, es decir, un año después de la muerte de Antonio Salieri, cuando los rumores sobre su responsabilidad en la muerte de Mozart aún se encontraban circulando (Borowitz, 2005: 140)

${ }^{5}$ Dice Derrida: «La différance es lo que hace que el movimiento de la significación no sea posible más que si cada elemento llamado "presente", que aparece en la escena de la presencia, se relaciona con otra cosa, guardando en sí la marca [marque] del elemento pasado y dejándose ya hundir por la marca [marque] de su relación con el elemento futuro, no relacionándose la marca [trace] menos con lo que se llama el futuro que con lo que se llama el pasado, y constituyendo lo que se llama el presente por esta misma relación con lo que no es él: no es absolutamente, es decir, ni siquiera un pasado o un futuro como presentes modificados».

${ }^{6}$ Dado que no dominamos la lengua rusa del original, hemos optado por consultar diferentes traducciones de la obra al español y al inglés. No obstante, para las citas, en este caso, seguimos una sola a modo de ejemplo.

${ }^{7}$ Dice Salieri en la obra de Pushkin: «No hay derecho: / el don sublime, la sagrada llama / no son premio del rezo, la fatiga, / los sacrificios, el trabajo duro. / No es justo, no lo es, que el don, la llama / iluminen radiantes la cabeza / de un loco, un libertino... ¿Mozart, Mozart?» (Pushkin, 1942).
} 
Una diferencia significativa entre ambas piezas es el rol del italiano en la muerte de Mozart: en Pushkin, Salieri envenena a su rival. En cambio, en todas las reescrituras de Shaffer, Salieri jamás asesina a su rival. Sí puede decirse que es el autor de su desgracia; también que luego de la versión 1 (1980), el italiano se involucra más directamente en el acoso del compositor disfrazándose como el mensajero enmascarado que asedia a Mozart, cuando antes el mensajero era un sirviente de Salieri (Shaffer, 2001b: XIX). Resulta aún más relevante la decisión de Shaffer de no convertirlo en el autor directo de esa muerte: Mozart muere luego de un largo período de convalecencia, probablemente a causa de una cirrosis. Salieri, narrador de la obra, desea ser inculpado del homicidio para que su nombre recupere algo de la fama que ha perdido hacia el momento en que comienza a relatar al público los hechos 8 : «If not in fame, then in infamy» es la consigna del compositor que se mantiene en todas las versiones. Estas persistencias son tan significativas como los cambios para la crítica genética. En el prólogo de la última versión édita (2001b), Shaffer manifiesta abiertamente su negación a convertir a Salieri en asesino a pesar de todo el conjunto de cambios que introduce en el desenlace de la obra.

De este modo, esa elección sitúa al dramaturgo en una clara polémica con respecto al planteo de Pushkin. El segundo elige seguir —y contribuir a — la leyenda romántica. El primero, desde una visión más desencantada e iconoclasta ${ }^{9}$, elige hacer a Salieri víctima de su propio complot para destruir a su rival, complot que paradójicamente termina por agrandar la figura de Mozart. Se dirá que Shaffer se muestra más próximo a un planteo existencialista, como señala Jorge Dubatti (2013), propio de la mirada desencantada e irónica que apareció en el teatro inglés de la posguerra (Innes, 2002; Dubatti, 2013). Ahora bien, si esta polémica es oculta o no en términos de Bajtín (1988: 300) nos devuelve a la pregunta: ¿en qué momento de la génesis aparecería este intertexto y de qué modo podemos localizarlo?

Lois (2001: 20; 2005b: 91) señala que la etapa conocida como «exogénesis» es aquella en la cual se daría la apropiación de los enunciados ajenos, frente a otra etapa denominada «endogénesis». No obstante, como el concepto de escritura del geneticismo es recursivo, estas etapas no son necesariamente dos instancias perfectamente separables ni se suceden cronológicamente en ese orden. En cuanto a la exogénesis de Amadeus, podemos señalar algunos puntos relevantes:

a) Shaffer además de ser un destacado dramaturgo en su país ha participado en ocasiones como crítico musical en la revista Time and Tide (MacMurraughKavanagh, 1998: 7) y es un erudito en la vida de Wolfgang A. Mozart.

b) Si el autor, tal y como manifiesta, desconocía la obra de Pushkin, resulta factible que haya conocido la ópera de Rimsky-Kosakov, que es bastante popular en el ámbito lírico; en este sentido, desde el geneticismo miraríamos la biografía del autor para establecer la intertextualidad probable.

\footnotetext{
${ }^{8}$ Recordemos que la diégesis de la obra se inicia en la noche en que Salieri planea suicidarse y, a partir de allí, se entrelaza con una segunda línea temporal de flashbacks.

${ }^{9}$ Esta visión puede rastrearse en otras obras del autor. Véase Lai (1989).
} 
c) Por otra parte, si Shaffer no conocía estos posibles intertextos, es probable que los haya conocido luego, una vez popularizada su versión de la vida del músico austríaco y que a partir de ese momento, la polémica fuese manifiesta.

d) Uno de los actores de la primera puesta en escena del drama, Simon Callow, en una columna publicada en The Guardian manifiesta conocer las obras de Pushkin, Rimsky-Korsakov, y sus diferencias con el texto del dramaturgo inglés, así como la constante presencia del Shaffer en los ensayos su conversación con él y otros actores (Callow, 2006) ${ }^{10}$. Por lo tanto, aquí hay un posible punto de contacto que avalaría la presencia de la intertextualidad.

e) Peter Hall, director de la primeras dos grandes puestas de Amadeus había realizado el montaje de varias óperas de Mozart. Cabe pensar que conociera la ópera de Rimsky-Kosakov o la pieza de Pushkin.

De este modo, tenemos un rango de posibilidades que permite pensar el contacto y el ingreso de Pushkin como enunciado-intertexto polémico a la génesis de Amadeus. El rango cronológico resulta amplio, pero antes que determinar una fecha precisa lo que interesa es determinar su presencia efectiva y, diría Derrida, la presencia atada a la diferencia semiológica (1998a: 47). Más importante aún, a partir de este momento, releemos en forma retrospectiva las reescrituras en Shaffer frente a Pushkin: el empecinamiento en evitar que Salieri asesine a Mozart, a pesar de que entre las versiones 2 y 4 se registren cambios en la actitud de Salieri como el arrepentimiento o la duda (Shaffer, 2001b: XXVI), se resignifica en función de una polémica oculta con el Mozart y Salieri. Como comenta Shaffer, en la versión 2 y en la 3 sintió demasiado presente la fuerza del melodrama y de los elementos góticos, por lo cual en versiones posteriores decidió «rebajar» la maldad inquebrantable de Salieri (Shaffer, 2001b: XXI; Demeer, 1997) y darle momentos de duda y arrepentimiento más pronunciados. En este sentido, Shaffer trabaja la figura del compositor italiano de un modo que polemiza con la figura más monológica ${ }^{11}$ de Pushkin y con los «excesos» que Shaffer vería en el tratamiento romántico de Mozart $^{12}$.

Por otro lado, si en Pushkin Salieri considera a Mozart un «ángel» y hasta un «dios» que viene a mostrarnos sus canciones celestiales, Shaffer amplifica y explota esta veta teológica para transformar

\footnotetext{
${ }^{10}$ Citamos las declaraciones de Callow: «I had heard Rimsky-Korsakov's one-act opera Mozart and Salieri -a setting of the Pushkin drama inspired by the notion that Joseph II's court composer Salieri had poisoned Mozart. Pushkin's play is brief, dark, chilling, a simple if haunting tale of envy and rivalry. / Shaffer, I discovered, had taken this grim anecdote as a starting point for a vast meditation on the relationship between genius and talent, postulating a Salieri who was industrious, skilful and pious, driven to homicide by a Mozart who was foul-mouthed, feckless, infantile and effortlessly inspired» (Callow, 2006).

${ }^{11} \mathrm{Si}$ algo prueba la presencia de la ironía (del modo en que la define la teoría de la enunciación, es decir, como antífrasis) es precisamente la divergencia y la polifonía en el discurso de un personaje. En este caso, Salieri se nos muestra como un caldero de ebullición de sentimientos y opiniones a veces más confrontadas que en otras.

${ }^{12}$ En relación con esto, resulta pertinente recordar una anécdota en la cual Shaffer refiere críticas de Margaret Thatcher que él recibió en persona. La entonces Primer Ministro del Reino Unido sostuvo que un hombre que había escrito música como la de Mozart no podía andar por la vida haciendo chistes escatológicos del modo en que la pieza lo mostraba (Callow, 2006).
} 
Amadeus en un drama existencial de la relación entre dios y el hombre, la singularidad y la homogeneización como ha hecho en The Royal Hunt of the Sun y Equus (Dubatti, 2013; Innes, 2002: 483; Lai, 1989). Pushkin solo esboza al pasar esa situación y los motivos de Salieri resultan más terrenales: no se trata de una guerra trascendente contra el dios que le ha negado el talento como en Shaffer (guerra de marcado tono paranoico e iconoclasta en Amadeus) sino de un asesinato perpetrado por envidia de fama. De este modo, Shaffer nos reenvía al texto de Pushkin para encontrar allí un estudio sobre la envidia creativa. Esto es significativo, dado que la obra de Pushkin encarna algunas ideas del genio romántico, movimiento para el cual la idea de originalidad resultaba clave. Amadeus, de este modo, afecta la lectura de Mozart y Salieri, nos permite releerla en clave del pensamiento de una época —algo cercano a la noción geneticista de «sociograma» (Lois, 2005a: 60) — y, a su vez, como una visión particular sobre las rivalidades entre modelos diferentes de artista.

Por otra parte, Shaffer establece diálogos con la del compositor austríaco en tanto Amadeus recurre a fragmentos de las piezas orquestales y óperas de Mozart. Aquí resulta relevante destacar cómo la firma Shaffer selecciona, interpreta y afecta el repertorio mozartiano para la construcción de su cosmovisión. Observemos algunos ejemplos.

Una de las lecturas más clásicas de Amadeus indaga en los aspectos psicoanalíticos de la obra; puntualmente en la relación entre Mozart y su padre, Leopold. Este último no aparece dentro de la diégesis de las piezas teatrales sino que resulta evocado por el discurso de los personajes y algunos recursos escénicos; en cambio, en el guión para la película de Milos Forman, Leopold es un personaje más. En ambos casos, se resalta su severidad y la tensión que genera en Mozart. No obstante, la intensidad de su presencia es un tanto distinta en las diferentes versiones, que muestran oscilaciones escriturales al respecto.

Shaffer se apropia de la música mozartiana para realizar un juego personal respecto de esta relación conflictiva: en todas sus versiones, se apropia del tema musical del comendador asesinado en Don Giovanni (representado por un furioso re menor en la obertura) para identificar a Leopold y, posteriormente, a un Salieri disfrazado para asemejarse a Leopold. Tenemos, en primera instancia, una célula musical que se resignifica hasta convertirse en un leitmotiv que atraviesa todas las variantes escritas de Amadeus y que construye su propia gramática musical. Del mismo modo, Shaffer selecciona fragmentos de las óperas que lleva al espacio del relato de Salieri. Así, se muestra la puesta en escena de Don Giovanni inmediatamente después del anuncio de la muerte de Mozart y se la superpone con el discurso de un Salieri que observa en esta obra una forma de destruir a su rival. Revisemos esta escena en la siguiente transcripción de las didascalias: 


\begin{tabular}{|c|c|c|}
\hline Versiones 1 y 2 & Versión 3 & Versión 4 \\
\hline $\begin{array}{l}\text { Scene } 9 \\
\text { [The two Grimm chords which open } \\
\text { ther overture to Don Giovanni sound } \\
\text { through the theater. Mozart seems to } \\
\text { quail under them, as he stares out } \\
\text { front. On the backdrop in the Light } \\
\text { Box appears a silohuette of a giant } \\
\text { black figure, in cloak and tricorne } \\
\text { hat. It extends its arms, menacingly } \\
\text { and engulfingly, towards its begger] } \\
\text { SALIERI: A father more acussing than } \\
\text { any in opera. So rose the figure of a } \\
\text { Guilty Libertine cast into Hell. } \\
\text { (Shaffer, 1980: } 129 ; 1984: 110 \text { ) }\end{array}$ & $\begin{array}{l}\text { 133 INT. AN OPERA HOUSE - } \\
\text { NIGHT - 1780's 133 } \\
\text { The second chord sounds. On stage } \\
\text { we see the huge figure of the } \\
\text { Commendatore in robes and helmet, } \\
\text { extending his arms and pointing in } \\
\text { accusation. } \\
\text { 133A INT. AN OPERA HOUSE - } \\
\text { NIGHT - 1780's 133A } \\
\text { The second chord sounds. } \\
\text { On stage we see a huge nailed fist } \\
\text { crash through the wall of a painted } \\
\text { dining room set. The giant armoured } \\
\text { statue of the COMMENDATORE } \\
\text { enters pointing his finger in } \\
\text { accusation at Don Giovanni who sits } \\
\text { at the supper table, staring - his } \\
\text { servant Leporello quaking with fear } \\
\text { under the table. } \\
\text { COMMENDATORE: (singing) } \\
\text { Don Giovanni! } \\
\text { The figure advances on the libertine. } \\
\text { We see Mozart conducting, pale and } \\
\text { deeply involved. Music fades down a } \\
\text { little. } \\
\text { OLD SALIERI: (V.O.) So rose the } \\
\text { dreadful ghost in his next and } \\
\text { blackest opera. There on the stage } \\
\text { stood the figure of a dead commander } \\
\text { calling out "Repent! Repent!" } \\
\text { (Shaffer, 1983) }\end{array}$ & $\begin{array}{l}\text { Scene } 9 \\
\text { [The two Grimm chords which open } \\
\text { ther overture to Don Giovanni sound } \\
\text { through the theater. Mozart seems to } \\
\text { quail under them, as he stares out } \\
\text { front. On the backdrop in the Light } \\
\text { Box appears a silohuette of a giant } \\
\text { black figure, in cloak and tricorne } \\
\text { hat. It extends its arms, menacingly } \\
\text { and engulfingly, towards its begger] } \\
\text { SALIERI: A father more acussing than } \\
\text { any in opera. So rose the figure of a } \\
\text { Guilty Libertine cast into Hell. } \\
\text { (Shaffer, 2001a: 83) }\end{array}$ \\
\hline
\end{tabular}

\section{CUADRO I}

El comendador es en la ópera original el padre de una mujer (Donna Anna) seducida por Don Giovanni/Don Juan; al defender a su hija, el comendador resulta muerto en un duelo con el seductor; posteriormente, reaparece en la obra en forma de la estatua que acosa al protagonista y termina arrastrándolo al infierno en una escena de marcados elementos góticos ${ }^{13}$. Salieri y Shaffer identifican al comendador con un Leopold que señala en forma acusadora la conducta del joven compositor. Es destacable también la oscilación en la descripción del comendador en estas cuatro versiones: mientras que en las piezas teatrales Shaffer lo despoja de su apariencia de estatua y acentúa los rasgos de los que Salieri se apropiará para acosar a Mozart posteriormente, en el guión presenta al mismo personaje conservando los rasgos de estatua del libreto de Lorenzo da Ponte para Don Giovanni y El burlador

\footnotetext{
${ }^{13}$ La presencia de elementos góticos en Amadeus es abordada en un trabajo en preparación titulado «Encapuchados y músicas nocturnas: los elementos góticos de Amadeus» en el marco de la realización de mi tesis. El motivo de la estatua que cobra vida y persigue venganza se encuentra presenta ya en la primera novela gótica, El castillo de Otranto, de Horace Walpole (1764).
} 
de Sevilla y convidado de piedra, texto de Tirso de Molina que inaugura la tradición literaria del Don Juan. A su vez, en el guión, la escenificación de la ópera se lleva a cabo inmediatamente después de que Constanze le anuncie a su esposo la muerte de Leopold; en las versiones teatrales, por otro lado, la ópera es mostrada luego de un discurso de Mozart en el cual llora y lamenta los disgustos causados a su padre. Nuevamente oscilan las intensidades entre las diferentes versiones, reafirmando las nociones de recursividad y proceso que la genética ha postulado.

Esta selección del fragmento culminante de la pieza lírica en cuestión muestra el sesgo de las elecciones de Shaffer: si bien Don Giovanni es una ópera tragicómica —y hasta catalogada por el propio Mozart histórico como opera buffa-, el autor de Amadeus elige presentarla como una tragedia debido a la porción que recorta. De este modo, al igual que con Pushkin, Shaffer nos devuelve al intertexto con una lectura personal: no polemiza con Mozart como sí lo hizo con el escritor ruso, pero sí realiza una selección y resignificación de la obra del austríaco.

Algo similar ocurre con Salieri. Shaffer también muestra una escena de su obra en la versión 3 de Amadeus: la apoteosis de Axur, rey de Ormus.

127 INT. OPERA HOUSE - NIGHT - 1780's 127

A performance of Salieri's grand opera, Axur: King of Ormus. Deafening applause from a crowded house. We see the reception of the aria which we saw Cavalieri singing on the stage near the start of the film. Cavalieri, in a mythological Persian costume, is bowing to the rapturous throng; below her is Salieri. We see the Emperor, Von Strack, Orsini-Rosenberg, Bonno and Von Swieten, all applauding. We hear great cries of «Salieri! Salieri!» and «Bravo!»

$[\ldots]$

129 INT. OPERA HOUSE - NIGHT - 1780's 129

Salieri conducting the last scene from Axur: King of Ormus. On stage we see a big scene of acclamation: the hero and heroine of the opera accepting the crown amidst the rejoicing of the people. The decor and costumes are mythological Persian. The music is utterly conventional and totally uninventive (Shaffer, 1983).

Teniendo en cuenta que Mozart ha despotricado previamente contra la ópera de tema mitológico, la selección operada por Shaffer aquí resuena con especial fuerza. Axur es también una ópera que no se clasifica unívocamente dentro del melodrama o el tono trágico; el Salieri histórico, a su vez, compuso también operas cómicas, hecho que Shaffer evita mencionar. El resultado es la construcción del personaje del compositor italiano en la cual se lo muestra como una figura severa, atado a valores clásicos opuestos a los de Mozart. Shaffer cierra esta idea con mayor intensidad en la indicación antes transcripta, la cual no esconde su valoración: «The music is utterly conventional and totally uninventive».

b) Lectura constelada de un problema: la risa. Para cerrar esta exposición, abordaremos un motivo de Amadeus que ya hemos mencionado: la risa. Si la bivocalidad de la palabra nos permite abordar la relación entre una obra y otra, es decir, un movimiento que a grandes rasgos podríamos denominar exogámico, haremos ahora un análisis entre las variantes escriturales de la propia obra. Al decir de Ferrer (2007), un pre-texto siempre se encuentra en polémica oculta con otros momentos de escritura; 
por lo tanto, la obra se muestra como un foro de discusiones que pone en evidencia el carácter dialógico de la lengua propuesto por Bajtín. Como hemos dicho más arriba, el rastreo de un problema textual dentro del proceso creativo se realizará desde una lectura constelada, ya que la misma nos permitirá encontrar puntos fluctuantes y borrar la direccionalidad que postula a la última textualización editada —en este caso, la de 2001 - , como la última palabra en el asunto.

Aun tratándose de una obra sobre un músico, es probable que el sonido más reconocible en Amadeus sea la molesta e insistente risa de Mozart. Esta funciona como otro leitmotiv, opuesto al de Don Giovanni al que hemos aludido. En este fragmento de Mozart y Salieri, Pushkin introduce dicho motivo en una escena en que ambos compositores observan a un músico que interpreta un fragmento de Don Giovanni.

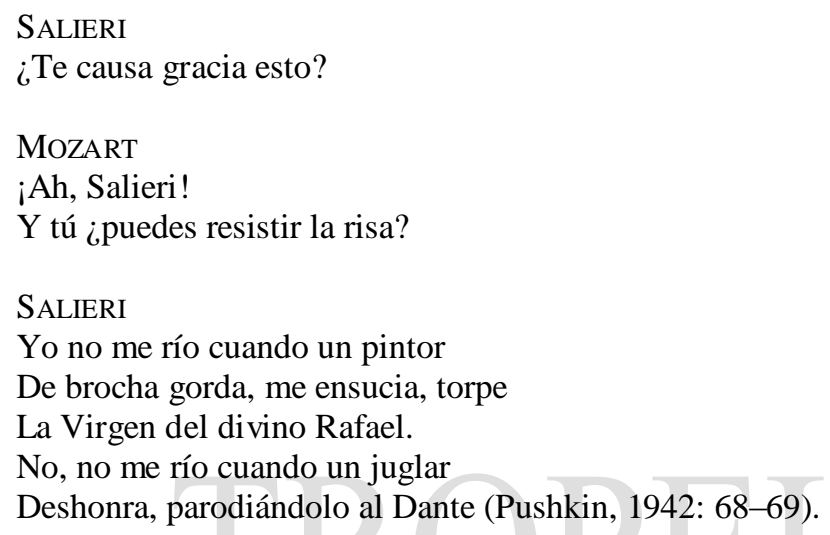

Su Salieri, desde una concepción de la elegancia y pulcritud musical, rechaza la labor del músico del bar. Aunque en Shaffer ocurra algo similar, la risa cobra diversos matices: la misma se muestra cercana al planteo que, nuevamente, Bajtín (1987) realiza sobre la función del carnaval, pues se muestra como un comportamiento que desafía el poder establecido, un mecanismo para trabar alianzas con un sector de la población y su cultura y una forma de defensa a la vez. De este modo, el dramaturgo se apropia de esta idea y la hace funcionar de diversos modos. A diferencia de lo que Henri Bergson establece acerca de la risa —un señalamiento de la transgresión del otro—, en Amadeus es en sí misma una transgresión. Observemos algunos ejemplos.

El primero corresponde a la escena presente en todas las versiones en la cual Mozart presenta el proyecto de su primera ópera, El rapto en el serallo, frente a la corte vienesa: 


\begin{tabular}{|c|c|c|}
\hline Versiones 1 у 2 & Versión 3 & Versión 4 \\
\hline \begin{tabular}{l}
\multicolumn{1}{c}{ Acto I } \\
Esc. 7 \\
MOZART: The story is really \\
amusing, Majesty. The whole plot \\
is set in a - [He giggles]. In a... \\
JOSEPH: [Eagerly]. Where? Where \\
is it set? \\
MOZART: It's- it's rather saucy, \\
Majesty! \\
JOSEPH: Yes, yes! Where? \\
MOZART: Well, it's actually set in a \\
seraglio. \\
JOSEPH: A what? \\
MOZART: A pasha's harem. [He \\
giggles wildly].
\end{tabular} & $\begin{array}{l}47 \text { INT. ROYAL PALACE GRAND } \\
\quad \text { SALON - DAY - 1780's } \\
\text { MOZART: It's actually quite } \\
\text { amusing, Majesty. It's set - the whole } \\
\text { thing is set in a - in a - } \\
\text { He stops short with a little giggle. } \\
\text { JOSEPH: Yes, where? } \\
\text { MOZART: In a Pasha's Harem, } \\
\text { Majesty. A Seraglio. } \\
\text { JOSEPH: Ah-ha. } \\
\text { ORSINI-ROSENBERG: You mean } \\
\text { in Turkey? } \\
\text { MOZART: Exactly. } \\
\text { ORSINI-ROSENBERG: Then why } \\
\text { especially does it have to be in } \\
\text { German? } \\
\text { MOZART: Well not especially. It can } \\
\text { be in Turkish, if you really want. I } \\
\text { don't care. } \\
\text { He giggles again. Orsini-Rosenberg } \\
\text { looks at him sourly. }\end{array}$ & \begin{tabular}{l}
\multicolumn{1}{c}{ Acto I } \\
Esc. 7 \\
MOZART: The story is really amusing, \\
Majesty. The whole plot is set in a - \\
[He giggles <sniggers>]. In a... \\
JOSEPH: [Eagerly]. Where? Where is it \\
set? \\
MOZART: It's-it's rather saucy, \\
Majesty! \\
JOSEPH: Yes, yes! Where? \\
MOZART: Well, it's actually set in a \\
seraglio. \\
JOSEPH: A what? \\
MOZART: A pasha's harem. He giggles \\
Wildly].
\end{tabular} \\
\hline
\end{tabular}

CUADRO II

Las reescrituras muestran cambios de intensidad en la risa de Mozart. El compositor conoce el carácter polémico de su ópera y pasa de risas más infantiles y agudas («giggle») a una más ladina y burlona («snigger»), la cual muestra el carácter desafiante del compositor frente a la ópera y el gusto oficial. Hacia el final de este fragmento, también hay cambios de intensidad: en sentido inverso, Mozart pasa de reír en forma descontrolada («giggle wildly») a no reír en la didascalia de la versión 4. Su silencio muestra cierta vacilación en su actitud desafiante.

Esa risa forma parte de un repertorio de elementos que trabajan la imagen del compositor desde el grotesco: el devenir niño de Mozart con sus juegos de palabras, el uso de vocabulario escatológico y la imitación de lo animal. A su vez, si bien el Salieri shafferiano retoma el carácter rígido del de Pushkin, así como su rol protagónico en la narración de los hechos, Shaffer agrega la ironía a su discurso. Este Salieri no escatimará en comentarios irónicos destinados al público, la auto-ironía con la que intenta trabar complicidades con el auditorio y un detalle grotesco: su desmedida pasión por los dulces que engulle.

Otro momento en destacable en la constelación de la risa es el rol de la misma en la escena final: en todas las versiones escénicas, Mozart y Salieri se confrontan y se descubre el rol del segundo en la caída en desgracia del primero. En la versión 3, en cambio, Salieri ayuda a Mozart a escribir los compases finales del Réquiem y Mozart jamás descubre el complot en su contra. Además, durante la escena de la escritura del réquiem, Mozart jamás ríe. ¿Por qué? Además de estar enfermo, el austríaco no se pone a la defensiva (una de las posibles funciones de su risa) con Salieri: lo reconoce como a un amigo y baja la guardia. En cambio, la risa con matiz defensivo aparece en las versiones restantes, 
cuando Mozart descubre los años de complot en su contra y, en una regresión infantil, responde a la oscura revelación con una serie de comportamientos infantiles que incluyen la burla, una risa negadora de la verdad y finalmente una regresión infantil. Esto se ve claramente en la versión 4, cuando Salieri intenta disculparse con Mozart y este lo toma como un chiste:

Mozart: Bravo! Bravissimo!... Signore, are you perhaps a Little tiddly? [Amused] I think you are!... Tiddly-widdly [Mock scolding] You had some before you came! I thought that might be so.

Salieri: [Desperate] Wolfgang, you must hear me now.

Mozart: [Giggling defensively] Tiddly-widdly-piddly!... Well, why not? It's not a cold night... And good performance! (Shaffer, 2001a:108)

Como vemos, en la versión 3, Salieri se libra del poder de la risa impidiendo que Amadeus ría. En las siguientes versiones no logra impedirlo. Vemos que no hay un trayecto unidireccional sino fluctuaciones. También hay recurrencias: Salieri, pese a su ironía, es prácticamente incapaz de reír al modo de Mozart, cuya risa lo une a otros: si Salieri ríe es por diferenciación o burla para con otro. El italiano sólo ríe en la versión 3 ante el atuendo turco de la cantante Katherina Cavallieri, y en alguna escena en la que intenta congraciarse con Mozart. Este último, en cambio, ríe al ver las parodias de su obra, ríe en relación con el vaudeville y la cultura popular con la que logra congraciarse en sus composiciones satíricas. Como dice una frase del comediante Victor Borge «Laughter is the shortest distance between two people».

\section{Conclusiones}

La visión geneticista propone aproximarse a la literatura con una mirada que problematiza su objeto de estudio al interesarse por las complejidades del proceso creativo. Como hemos visto, esta perspectiva puede brindar un marco teórico para abordar problemas como la intertextualidad en los estudios comparados y encontrar dentro de una misma obra la coexistencia de problemas que dan cuenta de las discusiones, afinidades y resignificaciones que se dan al interior de un texto. A diferencia de una concepción más tradicional del comparatismo que se aboque al cotejo de obras diferentes, la genética abre el texto literario y su carácter polifónico, revelando su riqueza pero también los desafíos metodológicos para su abordaje. Como diría Ferrer, «El escritor que tacha no es exactamente el mismo que escribe, el que redacta la segunda versión no es totalmente idéntico al responsable de la primera» (2007). Creemos que este enfoque puede aportar valiosas herramientas para el diseño de marcos teóricos y futuros estudios comparativos atentos a la complejidad de ese objeto que llamamos literatura.

\section{Bibliografía}

Angenot, M. (2010). El discurso social. Los límites históricos de lo pensable y lo decible. Buenos Aires, Siglo XXI.

BAJTín, M. (1987): La cultura popular en la Edad Media y el Renacimiento. Madrid, Alianza. 
(1988): «La palabra en Dostoievski», en Problemas de la poética de Dostoievski. México, Fondo de Cultura Económica, pp. 253-374.

Bergson, H. (2011): La risa. Buenos Aires, Godot.

Borowitz, A. (2005): Terrorism for Self-glorification: the Herostratos Syndrome. Kent, Kent University Press.

Callow, S. (2006): «It was the part of a lifetime», The Guardian (26-8-2006), en http://www.theguardian.com/stage/2006/aug/29/theatre (última consulta, 31-12-2015).

Chartier, R. (2006): «Esbozo de una genealogía de la función de autor», Arte filosofia, 1, pp. 187198.

DeEMER, C. (1997): «Fine-Tuning Villainy. Salieri's Journey from Stage to Screen», Creative Screenwriting Winter, en http://www.ibiblio.org/cdeemer/amadeus.htm (última consulta, 31-122015).

Deleuze, G., y Guattari, F. (1988): «Rizoma», en Mil mesetas: Capitalismo y esquizofrenia. Valencia, Pre-Textos.

DERRIDA, J. (1998a): «La différance», en Márgenes de la filosofía. Madrid, Cátedra.

(1998b): «Firma, acontecimiento, contexto», en Márgenes de la filosofía. Madrid, Cátedra, pp. 347-372.

Dubatti, J. (2013): «Hacer pagar el dolor del absurdo», Tiempo argentino (26-6-2013), en http://tiempo.infonews.com/nota/105477/hacer-pagar-el-dolor-del-absurdo (última consulta, 31 12-2015).

FERRER, D. (2007): «Algunas observaciones sobre la pareja intertextualidad-génesis», en P. GIFFORD y M. SCHMID, coords., La création en acte. Devenir de la critique génétique. Amsterdam-New York, Rodopi, pp. 205-216 (traducción de Diego Joaquín Braquet para uso interno de la cátedra de Filología Hispánica, UNLP, 2013).

GoldCHLUK, G. (2009): «El archivo por venir, o el archivo como política de lectura», en Actas del VII Congreso Internacional Orbis Tertius de Teoría y Crítica Literaria. La Plata, Facultad de Humanidades y Ciencias de la Educación, en http://citclot.fahce.unlp.edu.ar/viicongreso/actas-2009/Goldchluk.pdf (última consulta, 31-12-2015).

(2010): «¿Dónde sucede la literatura? Libro, manuscrito y archivo en Manuel Puig y Mario Bellatin», El hilo de la fábula, 8/9, pp. 93-100.

Hall, P. (2001): «Introduction», en P. SHAFFER. Amadeus. Londres, Harper Perennial, pp. VII-XIII. HAMACHER, W. (2011): 95 tesis sobre la filología. Buenos Aires, Miño y Dávila.

HAY, L. (1996): «La escritura viva», Filología. Número especial dedicado a la Crítica Genética, Año XXVII, 1/2, pp. 5-22.

InNES, C. (2002): Modern British Drama: The Twentieth Century. Cambridge, Cambridge University Press.

LAI, F. (1989): Peter Shaffer's Dramatic Vision of the Failure of Society: a Study of The Royal Hunt of the Sun, Equus and Amadeus. Tesis de Maestría. Burnaby, Simon Fraser University. 
LoIs, É. (2001): Génesis de escritura y estudios culturales. Buenos Aires, Edicial.

(2005a): «De la filología a la genética textual: historia de los conceptos y de las prácticas», en

F. Colla, coord., Archivos: Cómo editar la literatura latinoamericana del siglo XX. Poitiers, Centre de Recherches Latino-Américaines, pp. 47-83.

(2005b): «Las distintas orientaciones hermenéuticas de la investigación geneticista», en F. COLLA, coord., Archivos: Cómo editar la literatura latinoamericana del siglo XX. Poitiers, Centre de Recherches Latino-Américaines, pp. 85-124.

MacMurraugh-Kavanagh, Madeleine (1998): Peter Shaffer: Theatre and Drama. Londres, Mcmillan.

MuÑIZ, A. (1989): «Notas de investigación sobre la literatura comparada», en Notas de investigación. México, en: http://www.filos.unam.mx/mis_archivos/u8/01_mun_iz.pdf (última consulta, 3112-2015).

Pushkin, A. (1942): El disparo. Mozart y Salieri. Festín durante la peste. Buenos Aires, Emecé.

RASIC, E. (2013): La creación del espacio poético en Momento de Simetría (1973) de Arturo Carrera y en sus manuscritos: Una constante formación de la materia. Tesina de Licenciatura en Letras. La Plata, Universidad Nacional de La Plata.

SÉRIOT, P. (2003): «Bajtín en contexto: diálogo de voces e hibridación de lenguas (el problema de los límites)», en B. VAuthier y P. M. CÁTEDra, eds., Mijaíl Bajtín en la encrucijada de la hermenéutica y las ciencias humanas. Salamanca: SEMYR, pp. 25-43

SHAFFER, P. (1980): Amadeus. Londres, André Deutsch. (1983): Amadeus: the screenplay. Revisión de diciembre, 1982, en http://www.screenplaysonline.de/screenplay/6 (última consulta, 31-12-2015). (1984): Amadeus. Londres, Harper Perennial.

- (2001a): Amadeus. Londres, Harper Perennial(2001b): «Amadeus: the final encounter», en Amadeus. Londres, Harper Perennial, pp. xvxxxiv.

Villalobos AlpíZar, I. (2003): «La noción de intertextualidad en Kristeva y Barthes», Revista de Filosofía de la Universidad de Costa Rica, 41/103, pp. 137-146

WelleK, R. (1976): Concepts of Criticism. New Haven-London, Yale University Press. 